\title{
Analysis of Vibration Influencing Factors of High Precision Cylindrical Roller Bearing by Poor Information (Part I : Theory)
}

\author{
Xintao Xia ${ }^{1, a}$, Bin Liu ${ }^{1, b^{*}}$, Lijun Zhang ${ }^{2, c}$ and Xiangfeng Chen ${ }^{1, d}$ \\ ${ }^{1}$ Mechatronical Engineering College, Henan University of Science and Technology, Luoyang \\ 471003, China \\ ${ }^{2}$ National Center for Materials Service Safety, University of Science and Technology Beijing, Beijing \\ 10083, China \\ axiaxt1957@163.com, 'blbin1992@foxmail.com, ${ }^{\mathrm{C} l j z h a n g @ u s t b . e d u . c n, ~}{ }^{\mathrm{d}}$ black_xfc@163.com
}

Keywords: High precision; Roller bearing; Poor information; Qualitative fusion; Correlation degree

\begin{abstract}
The vibration of high precision cylindrical roller bearing is affected by many factors, which belongs to the problem of poor information. This article based on the poor information system theory and the qualitative fusion principle, and a variety of methods are used to analyze the main factors that affect the vibration of high precision cylindrical roller bearings.
\end{abstract}

\section{Introduction}

The cylindrical roller bearing [1,2] is a kind of bearing with a line contact of roller and raceway. It has features of big load capacity, strong radial load carrying ability, small friction between roller and ring race, and can be used for high speed rotation. Because of its special structure, it plays an irreplaceable role in the motor, generator, gas turbine, machine tool spindle and so on [3, 4]. With the development of technology and the increasing demand of the main engine performance, the quality of cylindrical roller bearing has been put forward more and more high demand, and its vibration control has become one of the main problems which restrict the quality of this kind of bearing.

There are many factors affecting the bearing vibration [5-8]. This paper mainly considers the part parameters during the bearing production process. Bearing parts have many parameters, among which main factors must be targeted selected in order to effectively control the bearing vibration velocity. Considering the experimental cost, how to find out the most important factor from limited data is the most critical problem, which is a typical problem of poor information $[9,10]$.

\section{Qualitative Fusion Principle}

Qualitative Fusion. In the given domain $U$, we know the set of solutions obtained by $m$ methods

$$
\begin{aligned}
& H=\left(h_{1}, h_{2}, \cdots, h_{i}, \cdots, h_{m}\right) \\
& h_{i}=\left(h_{i 1}, h_{i 2}, \cdots, h_{i j}, \cdots, h_{i n}\right)
\end{aligned}
$$

Where $\mathrm{i}$ is the method number and $\mathrm{n}$ is the number of data.

If the " $\subseteq$ " is "attribute coincidence", then there must always be at least one set of elements from $\mathrm{H}$ in solution set $\mathrm{H}$ that is the final solution $\mathrm{h} 0$ satisfying criterion $\Theta$, which is expressed as

$$
h_{0}|\Theta| \text { From } H \subseteq H_{0}
$$

Wherein, H0 represents the set of truth values of the system property; ${ }^{\Theta}$ is represented under the $\Theta$ criteria; |From $\mathrm{H}$ represents the element from the solution set $\mathrm{H}$.

Because the system information has complex and diverse characteristics, it is possible to get different results by different methods, and even the results may be completely contradictory, so it is 
very important to extract useful information from them. If all the solutions are considered as a solution set $\mathrm{H}$, qualitative fusion means that the subset h0 with some consistency element is extracted from the solution set $\mathrm{H}$ as the final solution of the system under certain criteria.

Qualitative fusion has two meanings: one is qualitative, only to find a certain consistency of solution and no more complex calculations; two is the fusion, all solution sets are considered, all elements of the final solution are from the solution $\mathrm{H}$, and no other information is contained.

Application of Qualitative Fusion in Vibration Analysis. Take the number of influencing factors that may affect the vibration value of the rolling bearing as $\mathrm{N}$, and the symbol set of the influencing factor $\mathrm{X}$ is

$$
X=\left(X_{1}, X_{2}, \cdots, X_{i}, \cdots, X_{N}\right)
$$

Using $\mathrm{k}$ methods to analyze the experimental data, we can get the sort sequence of the influence degree of $k$ factors

$$
X_{l}^{k} \succ X_{l-1}^{k} \quad l=2,3, \cdots, N
$$

Wherein, $\succ$ represents better than.

The sort sequence matrix is

$$
X_{i}^{k}=\left[\begin{array}{c}
X^{1} \\
X^{2} \\
\vdots \\
X^{k} \\
\vdots \\
X^{m}
\end{array}\right]=\left[\begin{array}{cccccc}
X_{1}^{1} & X_{2}^{1} & \cdots & X_{i}^{1} & \cdots & X_{N}^{1} \\
X_{1}^{2} & X_{2}^{2} & \cdots & X_{i}^{2} & \cdots & X_{N}^{2} \\
\vdots & \vdots & \cdots & \vdots & \cdots & \vdots \\
X_{1}^{k} & X_{2}^{k} & \cdots & X_{i}^{k} & \cdots & X_{N}^{k} \\
\vdots & \vdots & \cdots & \vdots & \cdots & \vdots \\
X_{1}^{m} & X_{2}^{m} & \cdots & X_{i}^{m} & \cdots & X_{N}^{m}
\end{array}\right]_{m \times N}
$$

In the sorted sequence matrix, select the influence factors in the left of the $n \leq N$ as the solution set $\mathrm{H}$

$$
H=\left[\begin{array}{c}
h_{1} \\
h_{2} \\
\vdots \\
h_{k} \\
\vdots \\
h_{m}
\end{array}\right]^{T}=\left[\begin{array}{cccccc}
X_{1}^{1} & X_{2}^{1} & \cdots & X_{i}^{1} & \cdots & X_{n}^{1} \\
X_{1}^{2} & X_{2}^{2} & \cdots & X_{i}^{2} & \cdots & X_{n}^{2} \\
\vdots & \vdots & \cdots & \vdots & \cdots & \vdots \\
X_{1}^{k} & X_{2}^{k} & \cdots & X_{i}^{k} & \cdots & X_{n}^{k} \\
\vdots & \vdots & \cdots & \vdots & \cdots & \vdots \\
X_{1}^{m} & X_{2}^{m} & \cdots & X_{i}^{m} & \cdots & X_{n}^{m}
\end{array}\right]^{T}
$$

Then the final solution $\mathrm{h} 0$ is a set of factors in $\mathrm{H}$, ie

$$
h_{0}=\bigcap_{k=1}^{m} h_{k}=h_{1} \cap h_{2} \bigcap \cdots \bigcap h_{k} \cap \cdots \cap h_{m}
$$

If $h 0 \neq \Phi$, the qualitative fusion has a unique solution.

In the final solution $\mathrm{h} 0, \mathrm{z}$ influencing factors are all main factors with no particular order affecting the bearing vibration. The h0 can be expressed as follows after re-numbering and resorting.

$$
h_{0}=\left(x_{1}, x_{2}, \cdots x_{i}, \cdots x_{z}\right) \subset X
$$

Wherein, $1 \leq \mathrm{z} \leq \mathrm{n}, \mathrm{n} \in[0.3 \mathrm{~N}, \mathrm{~N}]$. It can be seen that there is no new information in the final solution. 


\section{Methods to Obtain Solution Set}

Define the data sequence of influencing factors as $\mathrm{Xi}$

$$
X_{i}=\left(x_{i}(1), x_{i}(2), \cdots, x_{i}(k), \cdots, x_{i}(n)\right)
$$

The data sequence of bearing vibration velocity value consists is $\mathrm{Yj}$

$$
Y_{j}=\left(y_{j}(1), y_{j}(2), \cdots, y_{j}(k), \cdots, y_{j}(n)\right)
$$

Wherein, $\mathrm{k}$ is the data number, and $\mathrm{k}=1,2, \ldots, \mathrm{n}$.

Gray Correlation Degree. The gray relational analysis mainly evaluates the association degree among the sequences by analyzing the similarity and distance of the geometric shapes of the data sequences.

The data sequences $\mathrm{Xi}$ and $\mathrm{Yj}$ are pretreated separately to obtain new data sequences

$$
\begin{aligned}
& X_{i}^{\prime}=\left(x_{i}^{\prime}(1), x_{i}^{\prime}(2), \cdots, x_{i}^{\prime}(k), \cdots, x_{i}^{\prime}(n)\right) \\
& Y_{j}^{\prime}=\left(y_{j}^{\prime}(1), y_{j}^{\prime}(2), \cdots, y_{j}^{\prime}(k), \cdots, y_{j}^{\prime}(n)\right)
\end{aligned}
$$

Wherein

$$
\begin{aligned}
& x_{i}^{\prime}(k)=x_{i}(k) K=x_{i}(k) / x_{i}(1) \\
& y_{j}^{\prime}(k)=y_{j}(k) K=y_{j}(k) / y_{j}(1)
\end{aligned}
$$

Define the absolute difference as

$$
\Delta_{i j}=\left|x_{i}^{\prime}(k)-y_{j}^{\prime}(k)\right|
$$

the minimum absolute difference as

$$
\Delta_{\min }=\min _{i} \min _{k}\left|x_{i}^{\prime}(k)-y_{j}^{\prime}(k)\right|
$$

and the maximum absolute difference as

$$
\Delta_{\max }=\max _{i} \max _{k}\left|x_{i}^{\prime}(k)-y_{j}^{\prime}(k)\right|
$$

The correlation coefficient of $\mathrm{Xi}$ and $\mathrm{Yj}$ is

$$
\xi_{i j}(k)=\frac{\Delta_{\min }+\Delta_{\max } \xi}{\Delta_{i j}+\Delta_{\max } \xi}
$$

The gray correlation degree of $\mathrm{Xi}$ and $\mathrm{Yj}$ is

$$
\gamma_{j i}=\gamma\left(X_{i}^{\prime}, Y_{j}^{\prime}\right)=\frac{1}{n} \sum_{k=1}^{n} \xi_{i j}(k)
$$

In the formula, $\xi$ is the resolution ratio, and $\xi \in(0,1]$, usually take $\xi \leq 0.5$.

Relative Correlation Degree. After initialization, the sequences of $\mathrm{Xi}$ and $\mathrm{Yj}$ are changed to be 


$$
\begin{aligned}
& X_{i}^{\prime}=\frac{X_{i}}{X_{i}(1)}=\left(x_{i}^{\prime}(1), x_{i}^{\prime}(2), \cdots, x_{i}^{\prime}(k), \cdots, x_{i}^{\prime}(n)\right) \\
& Y_{j}^{\prime}=\frac{Y_{j}}{Y_{j}(1)}=\left(y_{j}^{\prime}(1), y_{j}^{\prime}(2), \cdots, y_{j}^{\prime}(k), \cdots, y_{j}^{\prime}(n)\right)
\end{aligned}
$$

And then through the starting point zero processing, the original data sequences are changed into

$$
\begin{aligned}
& X_{i}^{0}=\left(x_{i}^{0}(1), x_{i}^{0}(2), \cdots, x_{i}^{0}(k), \cdots, x_{i}^{0}(n)\right) \\
& Y_{j}^{0}=\left(y_{j}^{0}(1), y_{j}^{0}(2), \cdots, y_{j}^{0}(k), \cdots, y_{j}^{0}(n)\right)
\end{aligned}
$$

wherein

$$
\begin{aligned}
& x_{i}^{0}(k)=x_{i}^{\prime}(k)-x_{i}^{\prime}(1) \\
& y_{j}^{0}(k)=y_{j}^{\prime}(k)-y_{j}^{\prime}(1)
\end{aligned}
$$

The relative correlation degree of $\mathrm{Xi}$ and $\mathrm{Yj}$ is defined as

$$
\varepsilon_{j i}=\frac{1+\left|S_{j}\right|+\left|s_{i}\right|}{1+\left|s_{j}\right|+\left|s_{i}\right|+\left|s_{j}-s_{i}\right|}
$$

Wherein

$$
\begin{aligned}
& \left|s_{i}\right|=\left|\sum_{k=2}^{n-1} x_{i}^{0}(k)+0.5 x_{i}^{0}(n)\right| \\
& \left|s_{j}\right|=\left|\sum_{k=2}^{n-1} y_{j}^{0}(k)+0.5 y_{j}^{0}(n)\right| \\
& \left|s_{j}-s_{i}\right|=\left|\sum_{k=2}^{n-1}\left(y_{j}^{0}(k)-x_{i}^{0}(k)\right)+0.5\left(y_{j}^{0}(n)-x_{i}^{0}(n)\right)\right|
\end{aligned}
$$

Absolute Correlation Degree. The raw data sequence is processed by the start point zero processing, the sequences of $X_{i}$ and $Y_{j}$ are changed to be

$$
\begin{aligned}
& X_{i}^{0}=\left(x_{i}^{0}(1), x_{i}^{0}(2), \cdots, x_{i}^{0}(k), \cdots, x_{i}^{0}(n)\right) \\
& Y_{j}^{0}=\left(y_{j}^{0}(1), y_{j}^{0}(2), \cdots, y_{j}^{0}(k), \cdots, y_{j}^{0}(n)\right)
\end{aligned}
$$

wherein

$$
\begin{aligned}
& x_{i}^{0}(k)=x_{i}(k)-x_{i}(1) \\
& y_{j}^{0}(k)=y_{j}(k)-y_{j}(1)
\end{aligned}
$$

The absolute correlation degree of $\mathrm{Xi}$ and $\mathrm{Yj}$ is defined as 


$$
e_{j i}=\frac{1+\left|s_{j}\right|+\left|s_{i}\right|}{1+\left|s_{j}\right|+\left|s_{i}\right|+\left|s_{j}-s_{i}\right|}
$$

Wherein

$$
\begin{aligned}
& \left|s_{i}\right|=\left|\sum_{k=2}^{n-1} x_{i}^{0}(k)+0.5 x_{i}^{0}(n)\right| \\
& \left|s_{j}\right|=\left|\sum_{k=2}^{n-1} y_{j}^{0}(k)+0.5 y_{j}^{0}(n)\right| \\
& \left|s_{j}-s_{i}\right|=\left|\sum_{k=2}^{n-1}\left(y_{j}^{0}(k)-x_{i}^{0}(k)\right)+0.5\left(y_{j}^{0}(n)-x_{i}^{0}(n)\right)\right|
\end{aligned}
$$

\section{Summary}

Information systems are complex and diverse, and there are many methods to reveal the characteristics of information systems. Because of the different emphases, the characteristics of each method can be different. The qualitative fusion is used to fuse the feature set of the information system of each method to take the common solution as the general feature information, and the obtained results have significant reliability and representativeness.

\section{Acknowledgements}

This project is supported by Natural Science Foundation of Henan Province of China (Grant No. 162300410065).

\section{References}

[1] Y. Tsumori, Y. Oya: U.S. Patent 7,712,966. (2010).

[2] U.A. Patel, and S.H. Upadhyay: Tribology Transactions, Vol. 60 (2017) No.2, p.284.

[3] R. Zimroz, W. Bartelmus, T. Barszcz and J. Urbanek: Mechanical Systems \& Signal Processing, Vol. 46 (2014) No.1, p.16.

[4] M.H. Evans: Materials Science and Technology, Vol. 28 (2012) No.1, p.3.

[5] N. Ghaisas, C.R. Wassgren and F. Sadeghi: Journal of Tribology, Vol. 126 (2004) No.4, p.681.

[6] P. Göncz, M. Drobne and S. Glodež: Engineering Failure Analysis, Vol. 32 (2013) No.4, p.44.

[7] X.T. Xia, Z.Y. Wang and H. Chang: Journal of Aerospace Power, Vol. 20 (2005) No.2, p.250. (In Chinese)

[8] C.M.C.G. Fernandes, R.C. Martins and J.H.O. Seabra: Tribology International, Vol. 58 (2013) No.2, p.47.

[9] X.T. Xia: Assessment of Rolling Bearing Quality Based on Poor Information (Science Press, China 2016).

[10]Y.Z. Xu and X.T. Xia: Bearing, (2016) No 8, p.27. (In Chinese) 